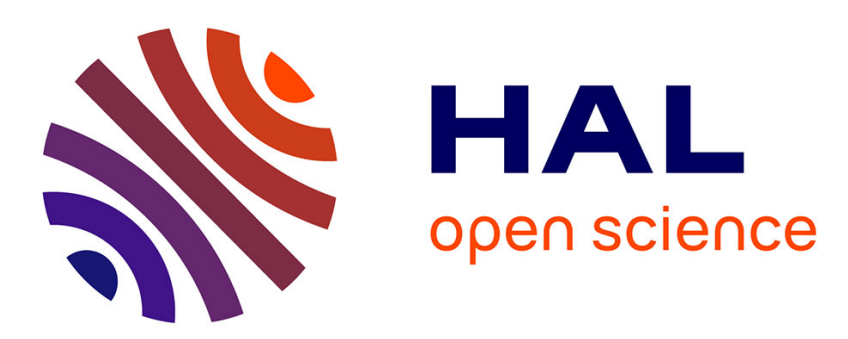

\title{
Fonctions des ornements à motifs géométriques dans la mise en page du texte des manuscrits grecs
}

\author{
Stavros Lazaris
}

\section{To cite this version:}

Stavros Lazaris. Fonctions des ornements à motifs géométriques dans la mise en page du texte des manuscrits grecs. KTÈMA Civilisations de l'Orient, de la Grèce et de Rome antiques, 2010, 35, pp.285-298. halshs-00576869

\section{HAL Id: halshs-00576869 \\ https://shs.hal.science/halshs-00576869}

Submitted on 15 Mar 2011

HAL is a multi-disciplinary open access archive for the deposit and dissemination of scientific research documents, whether they are published or not. The documents may come from teaching and research institutions in France or abroad, or from public or private research centers.
L'archive ouverte pluridisciplinaire $\mathbf{H A L}$, est destinée au dépôt et à la diffusion de documents scientifiques de niveau recherche, publiés ou non, émanant des établissements d'enseignement et de recherche français ou étrangers, des laboratoires publics ou privés. 
S. LAZARIS, «Fonctions des ornements à motifs géométriques dans la mise en page du texte des manuscrits grecs », Ktèma, 35 (2010), p. 285-298 


\section{Fonctions des ornements à motifs géométriques dans la mise en page du texte des manuscrits grecs}

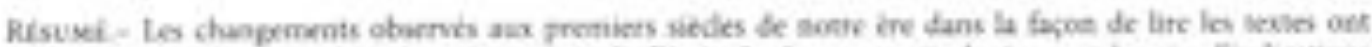

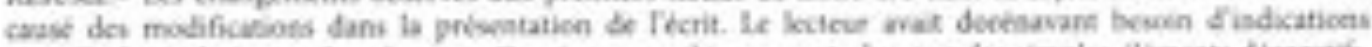

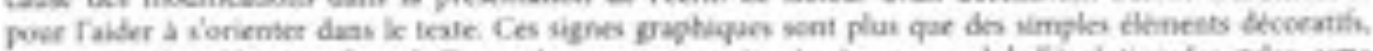

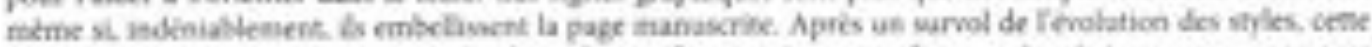

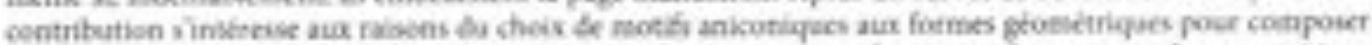

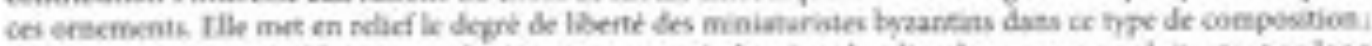

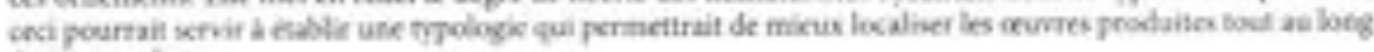
bu sowen is:

Anvikact, - During the first cesturies A. D, the way of readisg seats changed. and the affested the

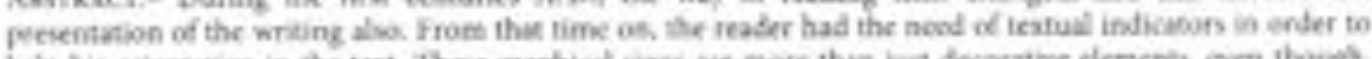

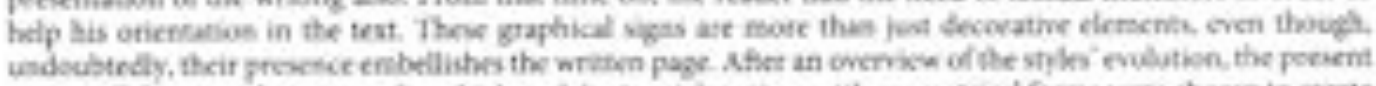
peper wil foces on the reasosa for which wod 'anicosic actives with goometrical forma wetr choses to create

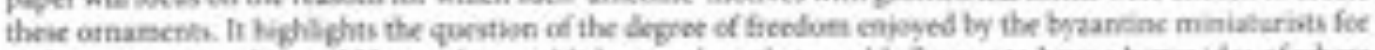

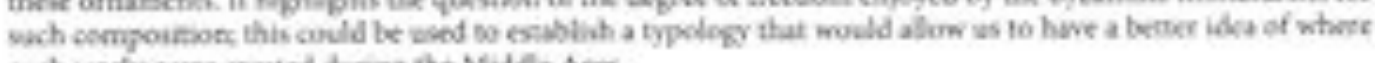
sach weiks were created daring the Midde Ages.

Des besolins appurus avec les beuleversements socioculturets scomecueifs a la venue du

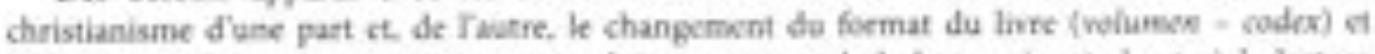
labandon de la isriptio contiewa om coeduit as pascoge de la lecture a woik haute a la locture a voris murmuate ou en silence. Cette nouvelle fagoe de life mais aussi la nócessite de trourcr

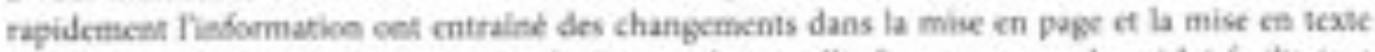
du livre-manascrit. L'utilisateur avait dorchavant besoin d'indicatcurs textuels quil lui facilitaient rorientation dans l'écrit en le hierarchisant. Toutefois, les exments participant à la mise en ondre du texte, si importants soient-ik, sont k plus wouvent claskis dans les etudes moderass comme etant de simples ornementa.

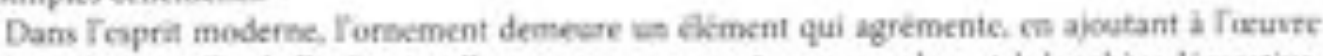
quelque chose qui l'enbellit. Partant, leomement col compriscomme elevant de la sphere décorative. sats autre ftelle fonction pour le lectrar médiéval que de faire plaisir a ws yeas. Cefte conception a vascite des réstions, dont la plas celebre demeure, encoce a se joer, Taphorieme d'A. Loos: - L'eenement est un crime $x$, Ea opposition fromale avec les escke ormermentaux du Jugendatil et de la Secession visneoise, il lance une ateaque virulente cuntre la productice dobyess surcharge et it 
assocke l'accumulation dornements as niveas le plus bas de la civilisation. Seloe les idtaux de Loos, ce şi n'a ni sens ni fonction nia pas dreet de cate dans une cruvfe. Si cette critieque pouvait etre fonder au togard dune arckilecture kelectique qui usait des ornements du passe comme d'autaat de citations savanten, elle a'ea repowail pas moins suf en malenendu. Hisooriquement. Fornement

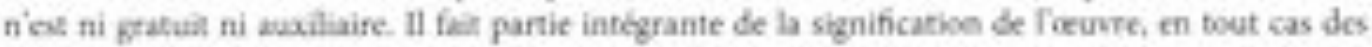
wovres qui sous intrescent ici: les manvescrits. Se pose alors la question de la pertinense de tenec utilise pour sesigner un element bont le role est decoratít

Cetie contribution sinteresse aux fonctions de ocs sornemenbs: dast le Inre-enanukcrit enlumind. qui constituc, pow ke Moyen Äge, la source la plus dirersifike des types omementaux mis en plack pour Torganisaboe du discours kcrit. Noas nous limiterons cependass ans eenements a motifs gbometriques, utilises pour geider le lecteur dafns sa lecture. T'analyserai dabond le nole de se tyge d'ormement en fappoet avec les changentsems dans la composition du manuscrit antique et medieval a Byrance, Suivront ensuite des consoderatioes pour expligeer lemplot de composition. a motifs getometriqaes aniconiques plutbs que d' ornements figuratifs.

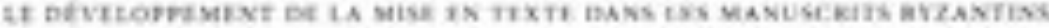

L'introduction de furnement dasis le lve mbdieval est etroitement associé a laménagement du discours ecrit", durant les premiers siccles de notre ene. Aupararant, le rapport de Thomme au livre-objet, mais aussi au teste et aax images gue renferme le livre etait bout autre. En cffet, justr'au II* siecle ap. I. C. dans le basin mediterrancen. ke livre arait communcment la forme d'un rouleau

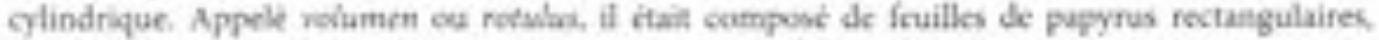
colles les unxs à la suite des autres par keur grand coec. $\lambda$ Tépoque aberandrine, en a noemalise la

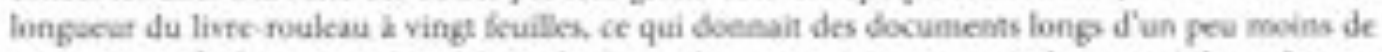
cissy metres. A chaque estzetmine enait placee une bagoette, quil permettait de soutenir le roukau ct de $k$ detouker de la main droite, randis que la gauche enrowlain. ta largeur du segment ouvert du couleau pouvait varier. bien qu'il restat

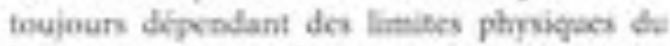
locteur: limites de lidcartertien de ses mains. limites du champ viveel humain.

Le iexte twail dispose en colonines etroibei paralleles. Cetre disposition, ains que la largeur des colonnes, waient êt rapidenent imposes par les limites du champ de perception. Sana distinction particalike porat les paragraphec. sans sfparation des mots. ni poncruation" ou toute autre subdivisicen, le texte ctail cectit dans une tcriture continuc, lascriprio confinia (fig-1).

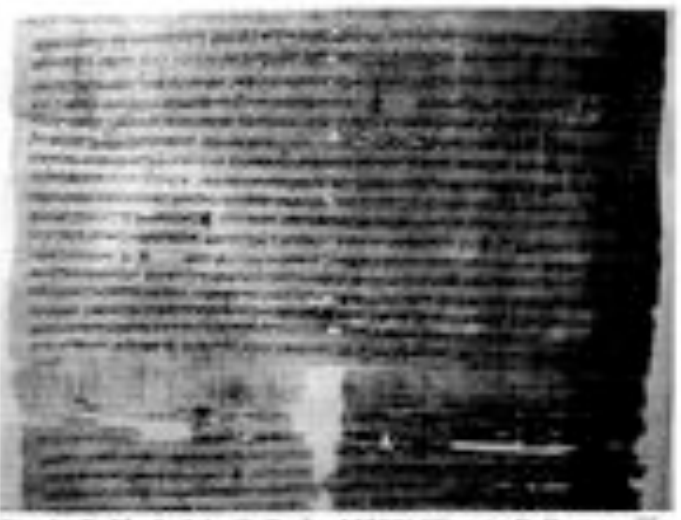

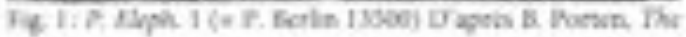

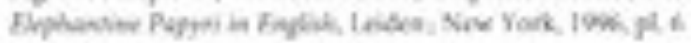

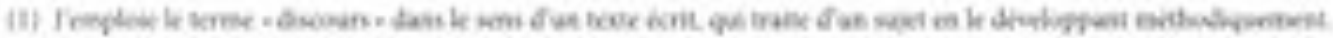

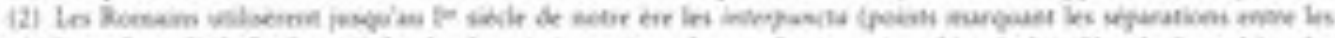

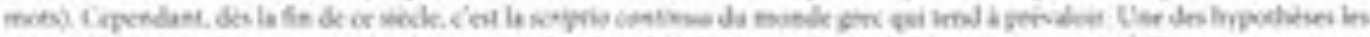

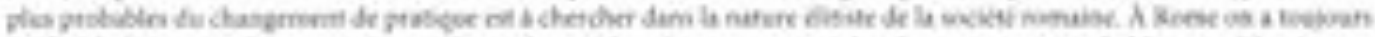

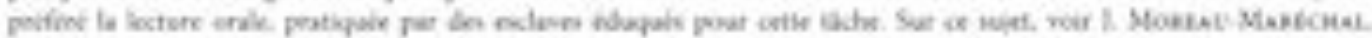

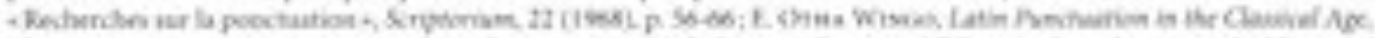

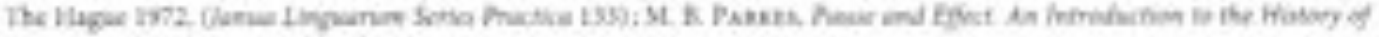

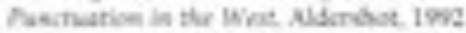




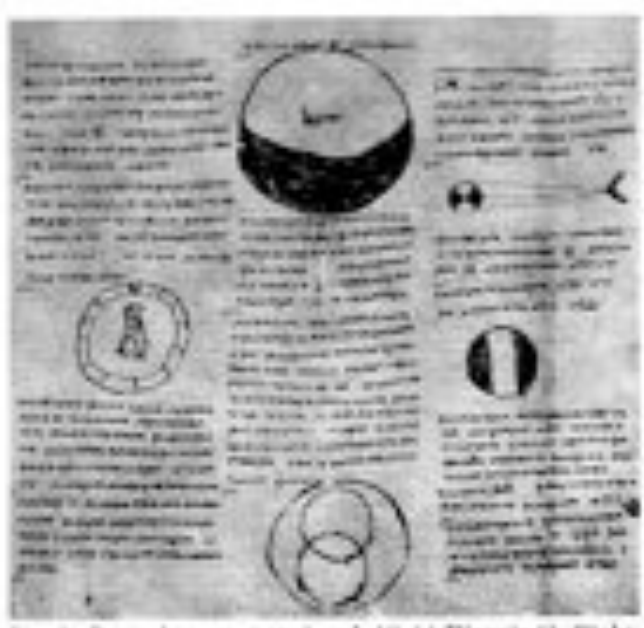

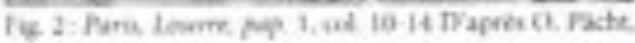

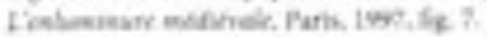

Lu seule interruption visuclle pouvail ètfe occasionnce par linastion d'images a Tinterieur de la colonne de texte. Cependant, comme en thetoighent los fares documents qui nows son parverauk, les tigures apparaisscnt sans aracune tçulariat: leur place semble kire uniquement determinte par les pasages al illustrer (frg 2). Lilles uiraient alors jone un role parement explicatif sans aucune iefention diatret la presentation de Thèr:

Leat prevence, pendamt une heture a haute voce risquait par aillewrs de power drs problimes au lectecar?. In effet. si une quakcoeque image venait internomgere cet afflus de letters majuscules justaposecs les anes a la vaite des autres sans la mo-dec separation, al est evident que la lecture et. far la mème, la comprebension du texte aaraient

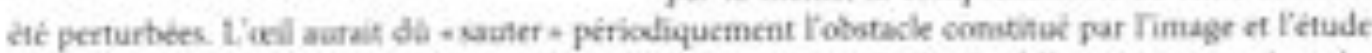
do teste serait passic au sccond plan (fig. 10). Cest ainsi que C. Nordenfalk noeait que when the ordinary scriptara coetimut hegins, decoeation becomes superflacus and stope =".

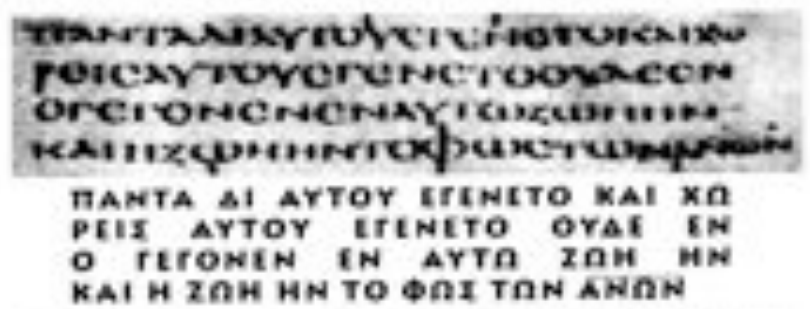

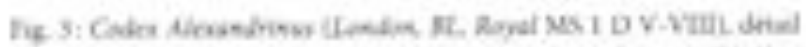

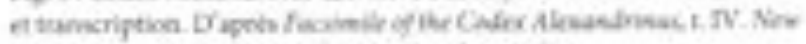

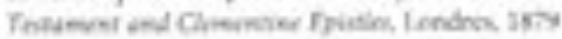

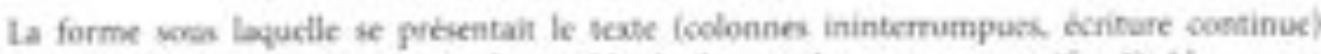

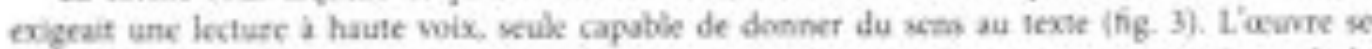
pretait plus à +entesdec = qu'a = voir = et ctaih, a priari, concue dans cette optique. issoe de la tradition ocale, la lecrure etas percue comme an acte de la vie ea societe ot se manifostait par la presesce of une personne lisant devant une asocmblec. La lecture solitaire ctait, toutefois, egalcment pratiquet. Ben que now anons qoxlques raecs témoignages de kecture silencieuse', ie lecteut

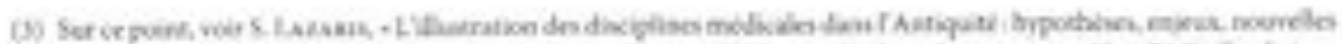

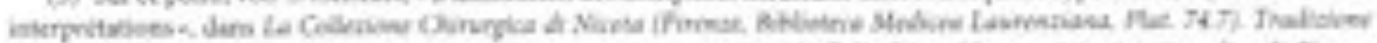

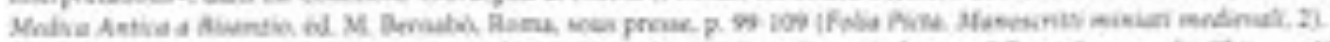

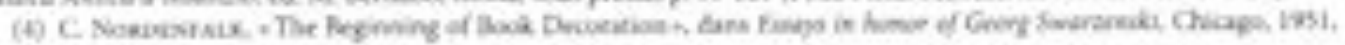
p. 12

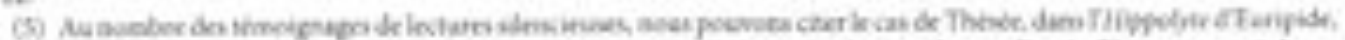

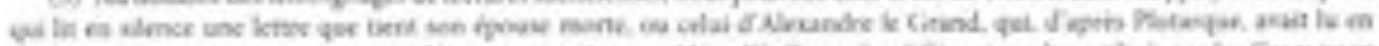

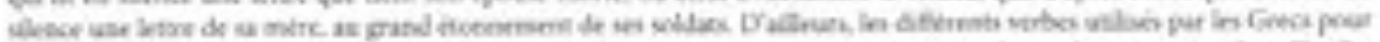

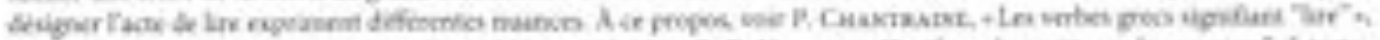

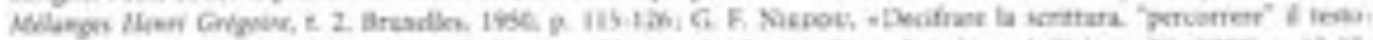

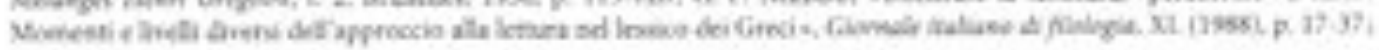




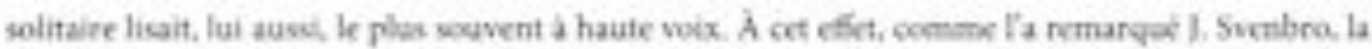

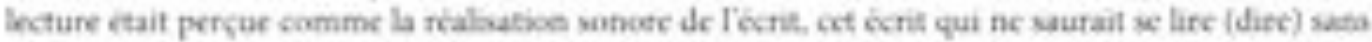
la vois du lectcur", Crest, en effet, par la bouche da lecteuf, aves won nthme et son semb, que l'terit pernail borme. Le teste deait aborde alors dans sa continuite, et la lecture, a haute voix, reproduisait cetle lintarité propoce al'expose oul.

L'absence d'un systeme de hierarchisation arain abors pour conscyuence de soumettre le lecteur a wne appreciation ininterrompae de Fruve. Ce dernier ne pouvait que difficilement revenir aur un pasoge particulier une fois le livre-ruukas rofermb. car asun signe ne lai permettait de se

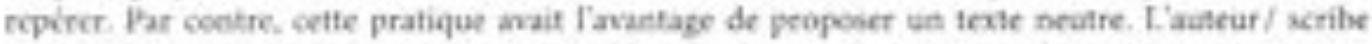

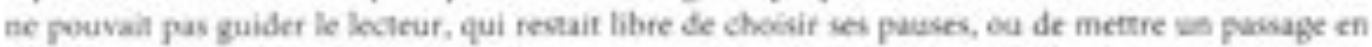
exergue plubibt qu'un autre. Seuk des changetsenss sociocultareb profonds arec le dkvioppement, entre autres, du chriatianisene et des evolutions techniques permiecat l'établissement d'un nouveau rapport entre Thomme es Tecrit, mals ausi entre lhomine et le live-objet, en remeetant en cause des habitudes siculaires.

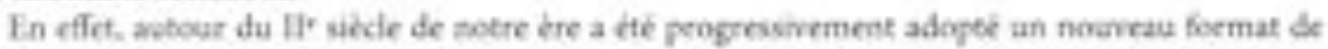
liver. Il s'agit du codex, qui fut d'aboed pletencite par kes chriticns?., Ainei que ke periciac R. Bugnall,

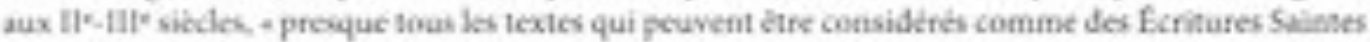
paraissem sous foeme de oudex =". Moins cher, puinque Iécrit occupe desoemais sysotmatiquement les deux còtés du suppoet". It codex a permis de faire face a une demande accrue de tentes. Sa forme derivait des rablettes de cire, qui etaient utilisetes dans le monde grec depais les XII- XIF- shcles

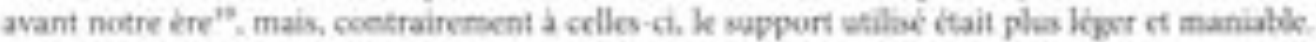

Ce nouveau format, qui reflete des mutatians sociales ot culturelles peofondec, a engendek ua chanpernent dam la notion meke de livre. Les bongs textes ctant sćpurtis suf plusiears fouleaus

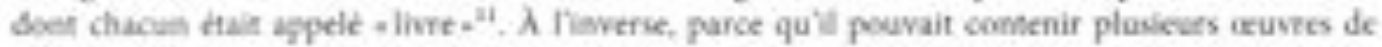
ditferentes largeurs, le codex vint a coincider wec la notios de - livre-oblyet-. Parce qu'be prut attacher un nombre variable de cahiers. le coviex possede, en effec. une capacibe beracoup plas grande que le roulcas. En raison du nombre accru de ses puges, on peut y reunir, dans wn wasque

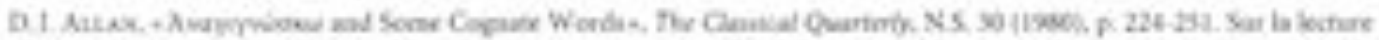

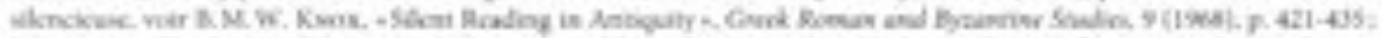

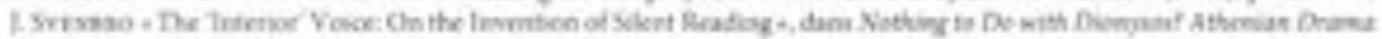

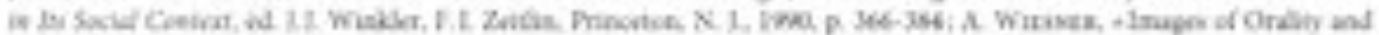

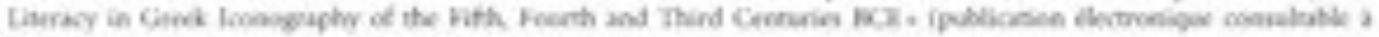

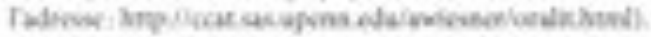

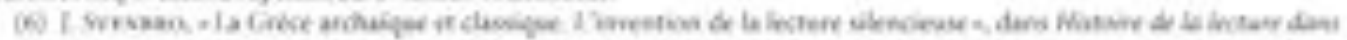

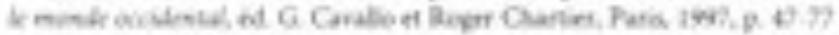

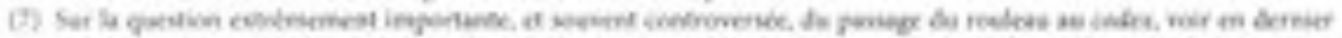

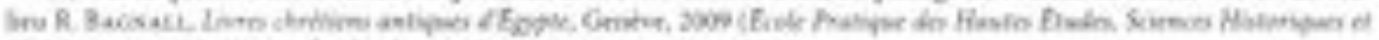

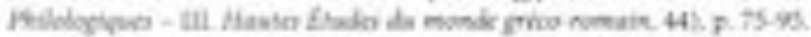

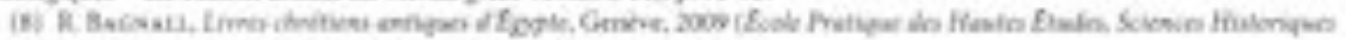

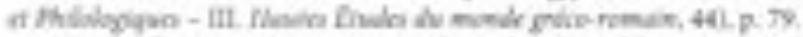

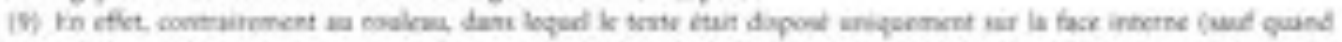

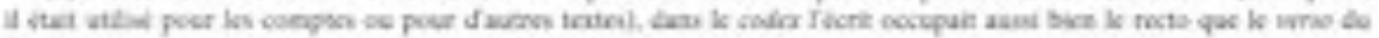
Anuilkt.

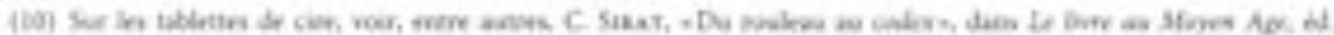

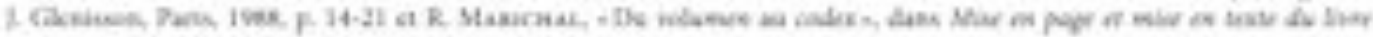

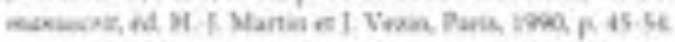

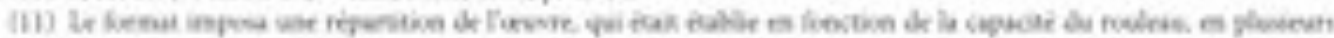

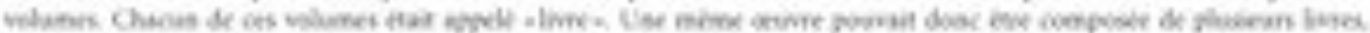

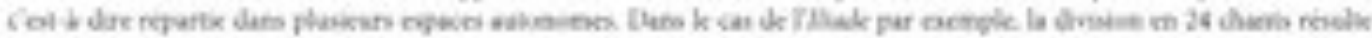

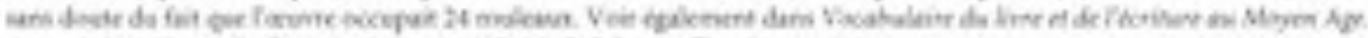

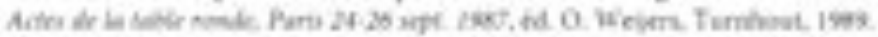




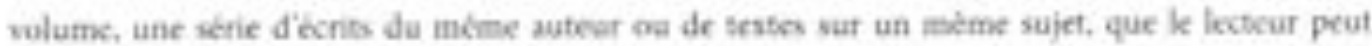
docknavant manipuler beasooup plus librement.

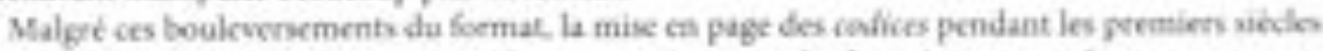
a imite celle du live-realeas. Cos codices, en presentant de deux a quatre sobonnes par page. reproduicaient limpteswions du scgment du rouleas labse vuvert pur son utilisateur ( $f_{\mathrm{B}}$-4). Toublois.

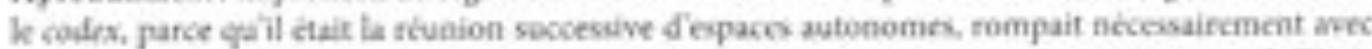

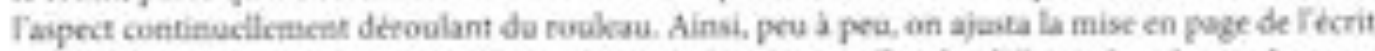
aux evuvelies possibilines offertes par se format. Lun de co effets fut dr tlargir la colonme de teste.

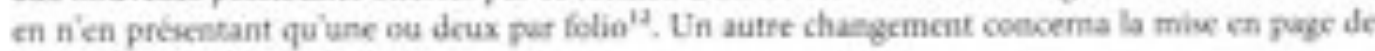
finuge" in tant quiktise autonome, le fectllet offrait. a cet kgard. de souvelles ponsibilites, les miniatues n'étaisnt plas uniquement insctries dans le corps du teste, mais pouvaicat occuper wois la moitie inféricure on saperticurs du folla woit la pleite page" On voit membe timbiement apparaltre quelques cssais dimages entre le corps du texte et la marge laticrale du

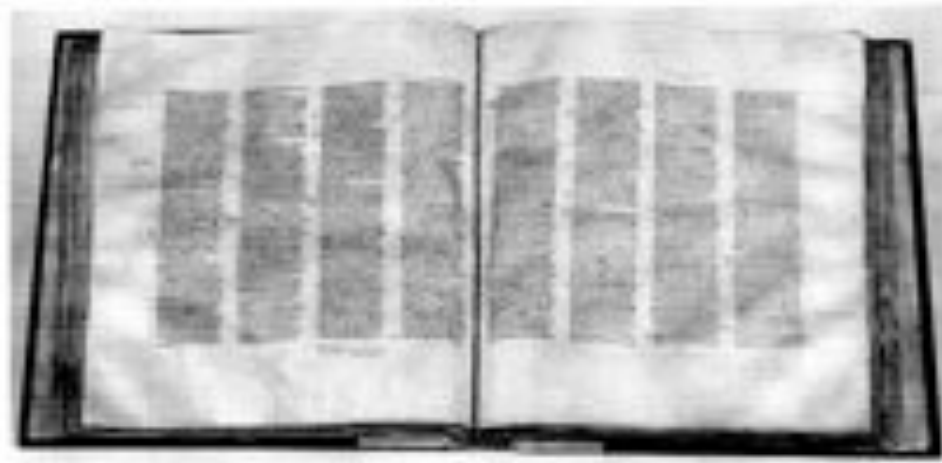

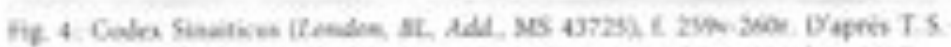

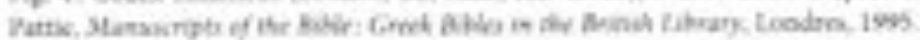
foliogin.

Cos difficrents types de mise en pace. rendas posubles grace a Tutitisation du nouveas foemat.

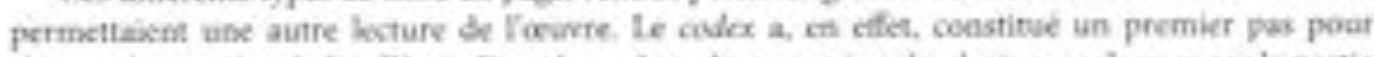

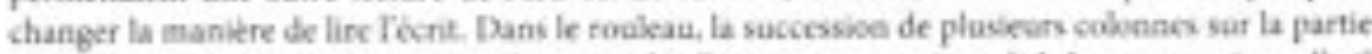

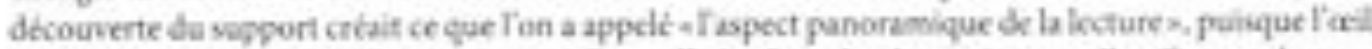
passait sans interrupsios d'une colonne a fuatre". Sur ke codex, la partie tesluclle efferte wa lecteut etain fonction de la taille de la page. Toutefois, tant que Tecriture contimae perdaratc, la kecture d haute vois feait quasi obligatoire pour bien wivir Is contene iestuel. 1es ecriss (scripta) restaient. en eflet, inertes tant que la voox ne lear denenait pas vie eat les transformant en mots (rerba), Ie premief cas pricis de lecture silencicuse nemonic aux Confessians de saint Augustin (VT, 3.3), qu seconnait de woir saint Ambroke pratigues une telle lectuec. Cette facon de lire corropondait a une

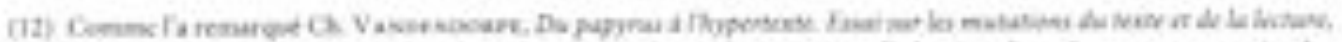

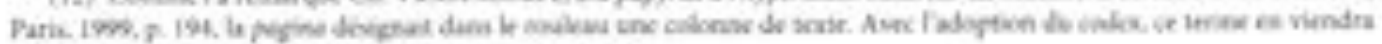

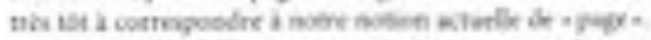

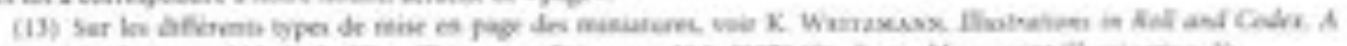

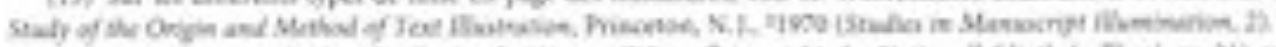

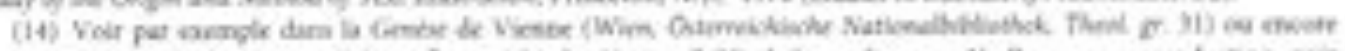

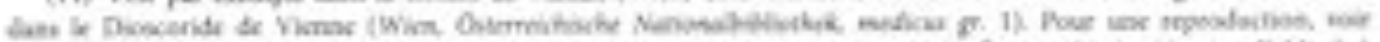

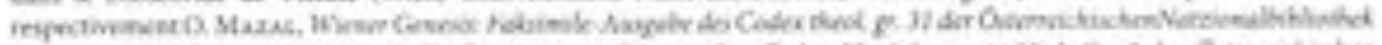

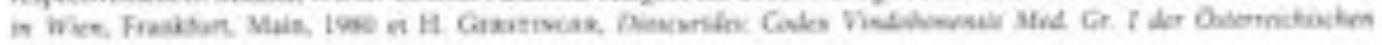

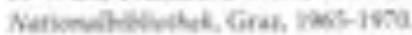

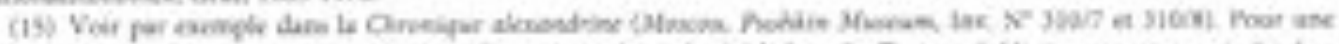

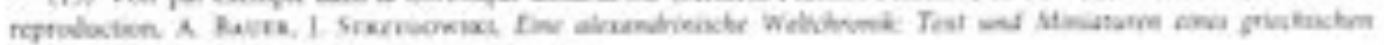

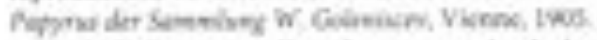

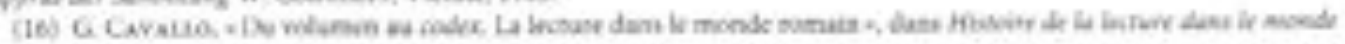

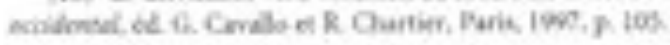


utilution souvelle da sexse qui se developpait Jams ks moastères, ou la lecture etait avant tout une invitation au recueiliement. Le texte, de surcrobk celui des ecrits sacres, devient effectrvement un objet de meditation qu'on lit attentivement posar sod-meme. de preierence en silence

Afin de facliter cette facon de lire, mais aussi pour permettre un achis rapide aux pasabes que Ton

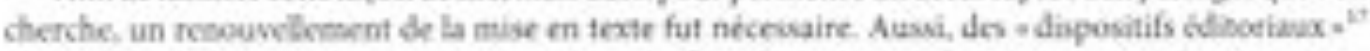

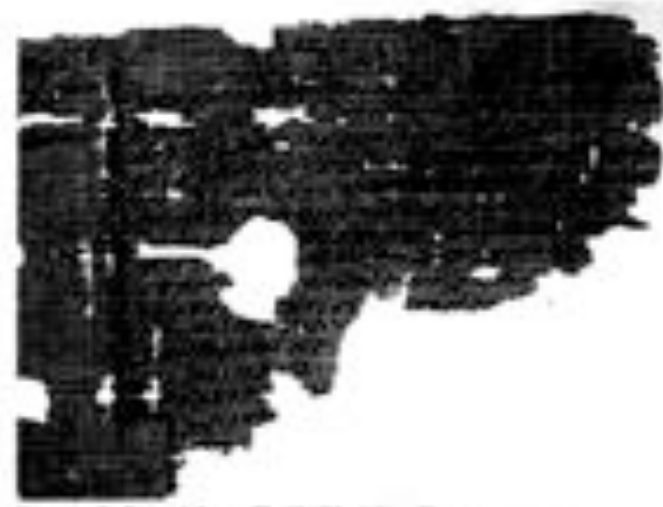

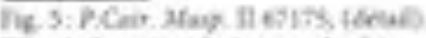

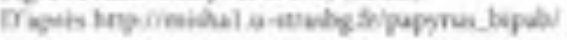

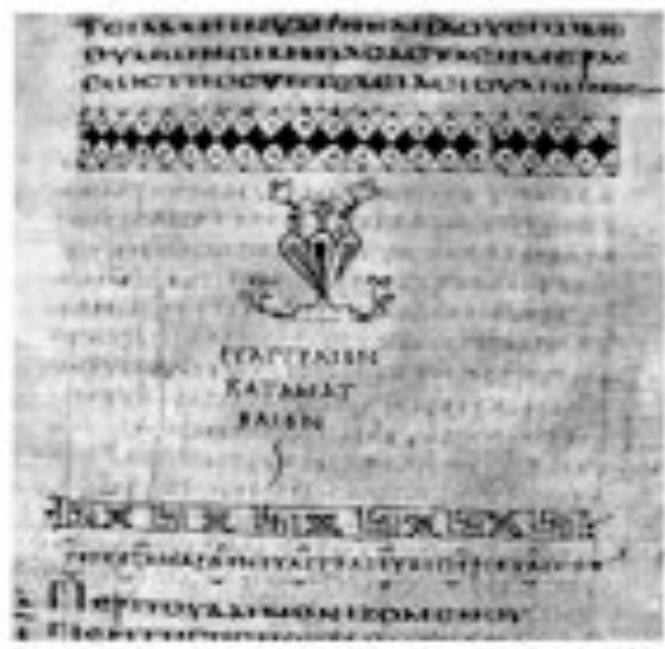

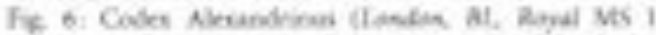

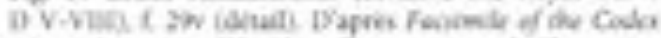

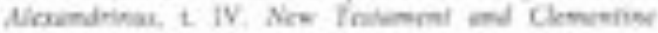

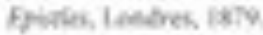

funt-ils peu a pere leur apparition; a commencet par les tieres et les foemules initiales et finales (incipir et acipin) qui signalent les siparabons entue les ecrits. La presentation du tcolce dans son ensemble commence peu a pru a se modifier en profondeur. Les potemiens ornements font awsi leur apparition pour distinguet visuellement, avec plus de clarte, des uniobs de sens de l'ecrit (hig. 5 ef 6). On augmente tgakement la taille des initiales et on procide sowvent à wn retrait (positif ou négatis) dans la marge, afin de mieux Nenaler le debut de chaque nouveau puragraphe. Par la vaite, on comttesece a utiliser une écriture minacule arex lettres invatiables, a stpaecr les mots a 1 anvif recours a la ponctuation pour edaifer la syatase". L"appretiatice de Powrre esait alors, pour beacoup, conditionnce par Fagencement de l'ecrit. M. Parkes explaque cette situation en écrivanl: cl'écri ctait desormais un langape visble cai allait difectement a liespeit par l'intermbdiaine de Toeils ${ }^{1 *}$. Le lecteor thait ains: guide pur la mise en tente et ce re'ctais plus par sa bouche que le discowrs prenait forme.

En imposant as beteur une compréhension determince du taske, ces changements techniques ont eu lavantage de faire perdurer la pensée de lauteur. The ont eu eqalenticas des fépercussions sur la deteonstration de la validioe du savoir scientifique. En effet, le format du colex, maís ausi les deterents signes graphiques cmployss et. plus tand. les index. Ins sommaines et les autres ovtils connexes, ent permicis une vérification relativement facile des réferences". Comme

(17) G. Caraus and, in bae

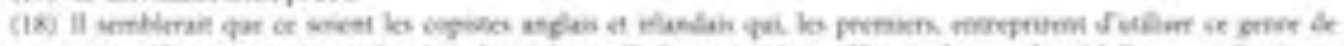

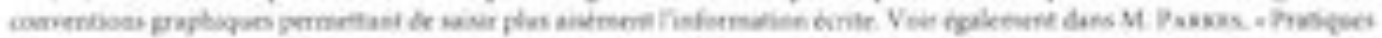

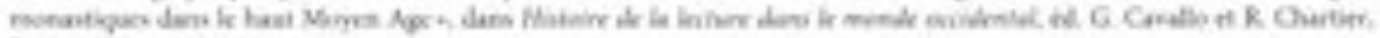
Parns, imot, p. 100.123

(19) M Psens, itu, p. 112

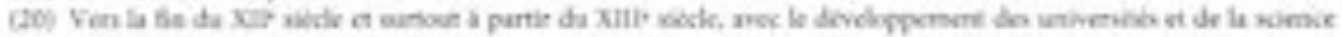

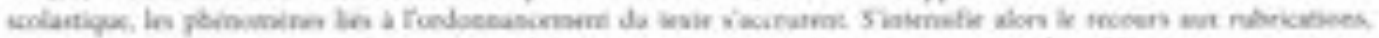

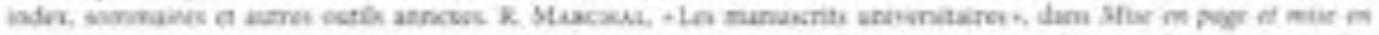

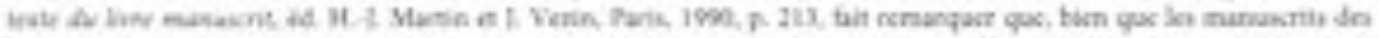




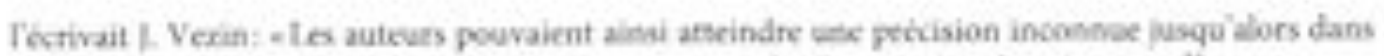
Tufilivationt de leurs sources, qu'ils citaient auparavant $k$ ples souvent de memoire.

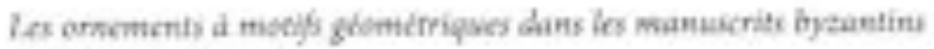

L'érolution des styles

Comme il a ete signale. Fornement apparait progressivemem aux IV: V. siecles et va suivre

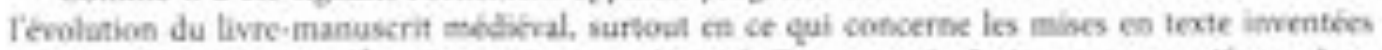

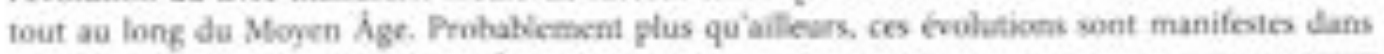
les tetracvangles byrantins, qui boement un carpus coberent es represuentatif". Malheureusememt de facoe ginerale, went conservis tiés peo de manasrits de l'epeque pre-sconoclaste et encore moins de manuscrits illustes (miniatures et $/$ ou ornements iconiques ou anticoniques\}. Au nombre do raros comples parvenus. saznabons le codex Alexwedrimus du W soble, qui contient plavieum motifs ornementaus, qui evolucront par la wite (fig. 6). Fn ce quit conoerne les tetrackangiks de cetie çoque, nous ne disposons que de fragments comportant des motifs poumetriques, dont le Landon,

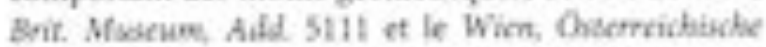

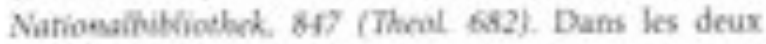
remoins, los careons d'Fasibe el sa lestre a Carpien sont placks dans une stracture architectarale a cotomne Et froeboe. les colonnes de chiffres sont encadróes de colonnettes couronndes de tympans ou de pigsens. les mocifs sont ceux que ron retrouneta pendant les siecles raivants: des trawes ou des tiges wax courbes irregulikeres des riguas, des lonanges, des mootifs condiforeses (hip. 7 ct 14). Noas renocetrons f́galement des formules oeiginales qui ne scroot jos reprises, comme cette cowronne forme d'auróoles juxtuposbes qui entoure le titre des carnoss de concordance dans Fevangile de Rossano (Rosidsa, Muse Arcivescovile.

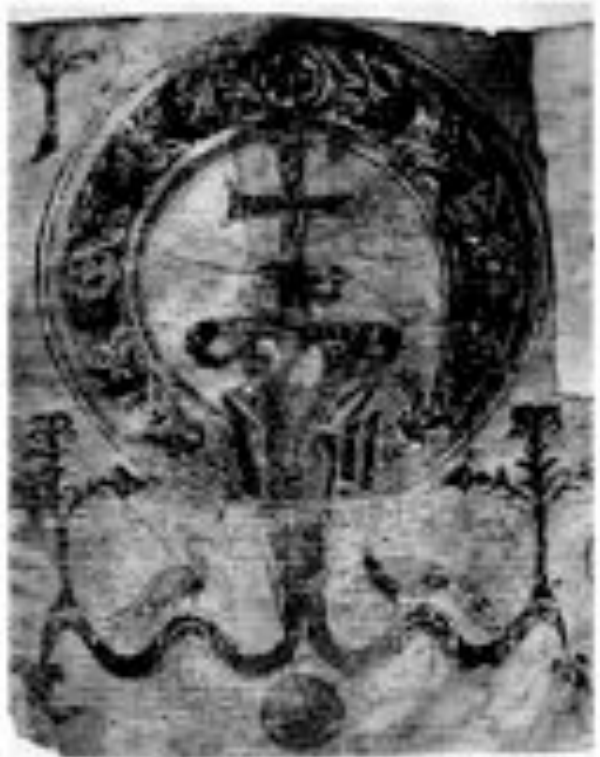

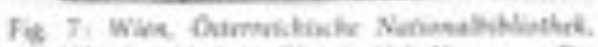

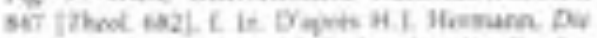

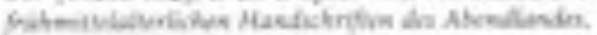

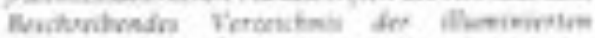

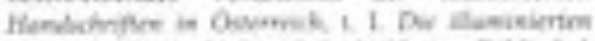

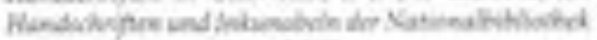
in Wike. Leripas 1v2x fal vill codex Purpuress, $(.5)^{20}$,

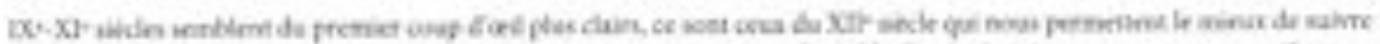

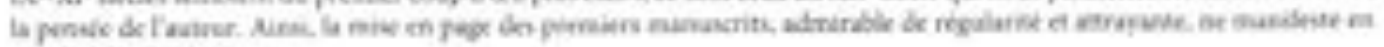

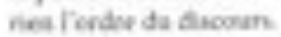

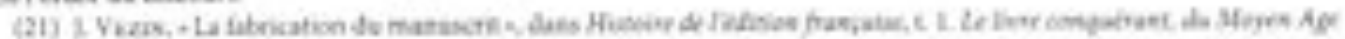

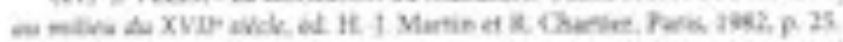

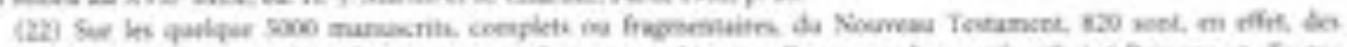

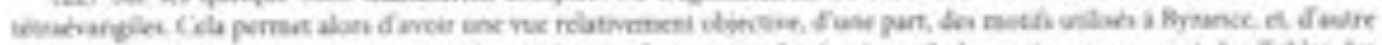

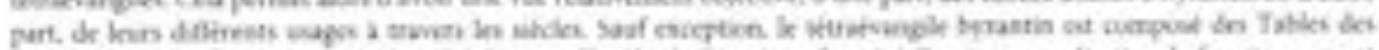

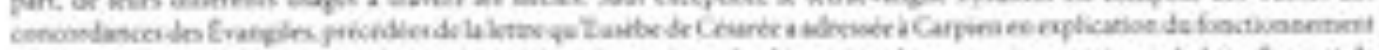

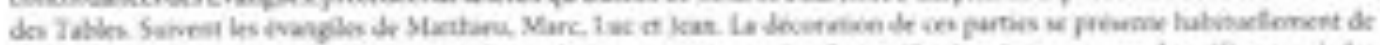

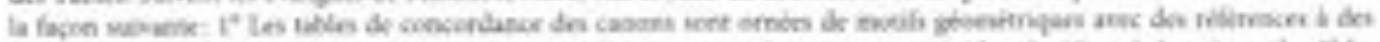

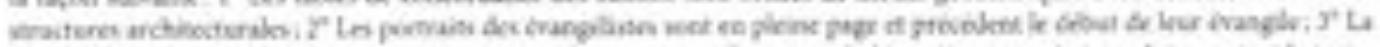

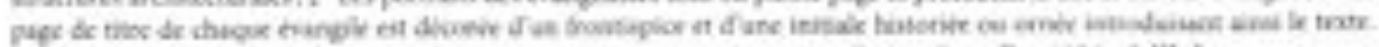

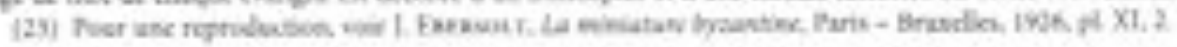


Ciot apeśs ficonodlasme que la tendance ornementale s'affirme. Le plus asosen manuksit daté

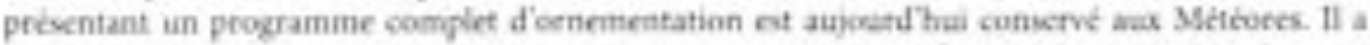

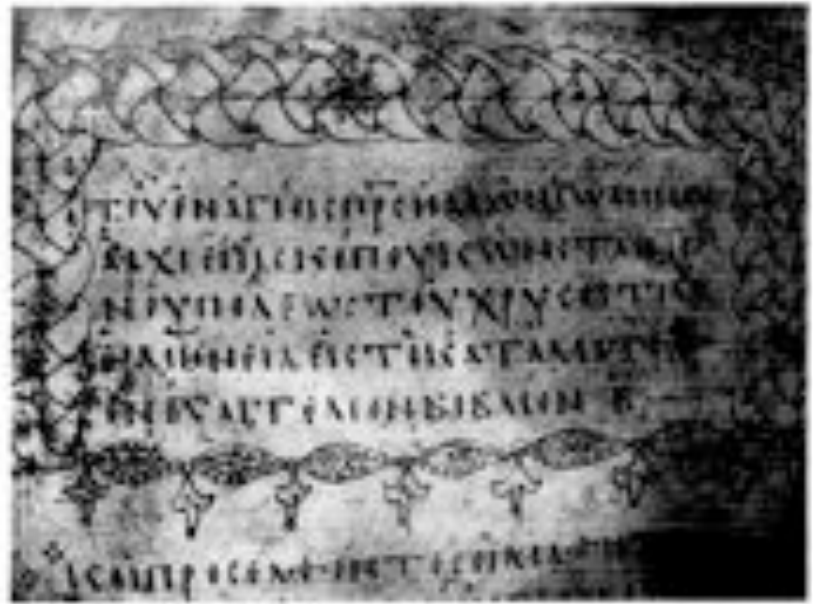

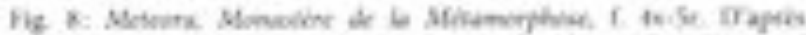

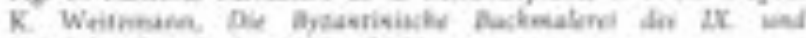
$x$. Jakutuedmbe, Beriin. 1935 at 200 cté contectionne en $862-863$ dans un monabietre de Bithynie (fig. o). Mais ke plus connu des codices illustres de cotte epoque est trés probubloment le Parie g. 510, qui somtient los Homdies de Grofvoire de Narianze, Ce volumineux mamakrit du $\mathrm{IX}^{*}$ sihcle pretiente les differentes spsiemes mis en place depuis l'investion da coulex et labandoe de la scriptio conninas pour strecturer le teste. Chaque homelie commence dacs on cadre a mocifs prometriquen. Les initiales, mais ause les miniateses,

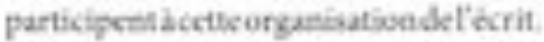
Nous rcmarquons mème differems types de busdeans et d'initiales qui correspondem a une gradation dans lindication des pascages importants" Ce phinosmatin de gradation par les motifs veilises = qui va de portig̨aes et de commencrements de prifaces richement peints jucqu'aus

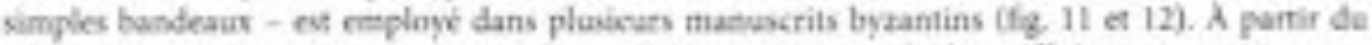

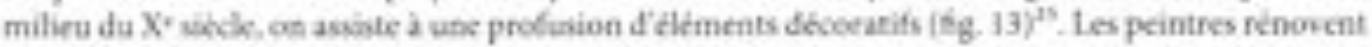

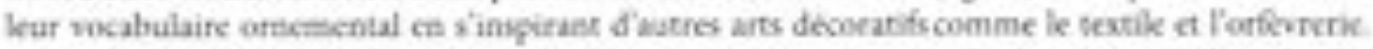
Les cndroits sescepebles de recevoif un decor se precisent et wont des bors rester inchangés. On asoite desormais a une codificarion des shèmas.

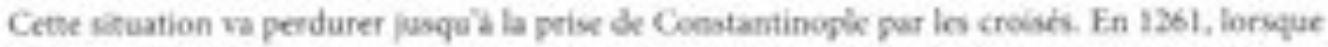
les Byzantins regagneront keur capitale, ils seont obligks de combler les lacuses ceeskcutives aux

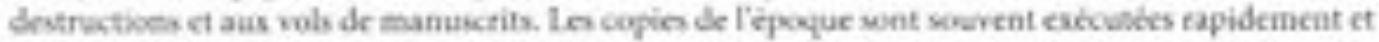
ks ornements n'eet plus la mëme place qu'aspuravant. On acklige d’ailieurs sowvent de les coppler

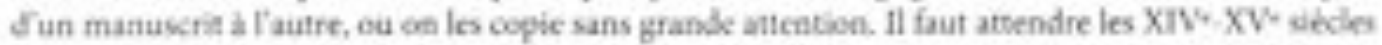
pour que les exnenteass retrouvem leur place dans les manuscrits. Nows sommes ki a la derniker feriode de la civilisation byzantine, mais ausa a une petriode caracterice par en important retlouveas culverel. On lit braucoep et on echange escece plas enire sirants byrantink, mais ausi arec les enudits latins. L'ornement, dans cette effervescence culturelk, fetrouve sa fonction de point de repere à l'imetrieur du teste. Le savoir prend désurmais unc autse place dats la sociéné medievale ef les ornements jouent es tüle sosenticl daes ce changement societal, gai est a la base meme du développement de la pensóe siemtifique esoderne. Liatroduction da plus simple amement contribue alors a l'organisation et a la petsentation de f'ecric, sipalant au lecteur une articulation. L'oraement devient an esement signifiant da contens, en permettant, d'un simple coup d'a't.

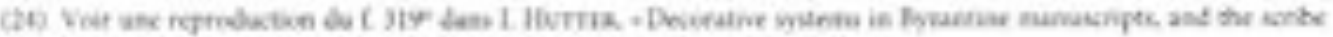

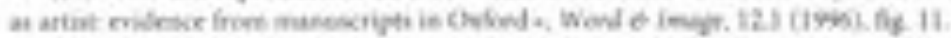

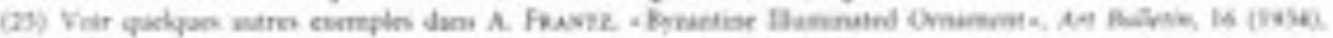

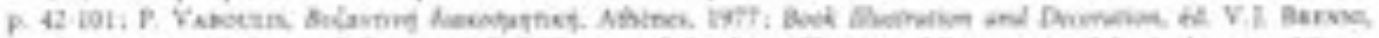

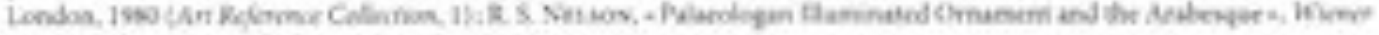

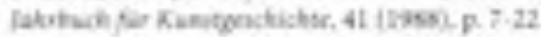


d'aperceveir la structure getnerale de Teruve. II particlpe abers activement a ube transmisoion hierazchisete du tente, en brisant Tunité de fecrit et en insistant, par des moyens visuels, sur cetaines de ses parties. In wa mot. il intellectualise le contcnest, La présece des ornements dans les manuscrits ne releve pas du simple plaisir estheticue.

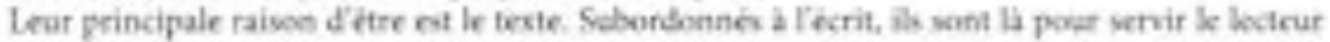
du manuscrif pedaest la lectere et non pas pour soffrit en spectacke. Ce contal niest pas tovaveau, mais il gagne à ête sodigne si Ton veut apprehender use question qui, elk, semble avoir ced peu itualice. Il sapit de savoir pourquovi libustrateuf a eu fecours a des motifs peometriques plutot qu'a des oencments fizuratifs pour scander le texte. Ceste question se trowe as corur de nos mbanismos cognirifs d'apprehension du reel et les quelques considerations que je vas prohenter ici wont los premieres ebasches d'une recherche

\section{Des motifs poar gaider, non pour perturber}

Ceal justcmes parce que ces bandeasax, colonines et autres onements ont pour nole de guider la kectuec qu'lis sont giometriques. Leur emploi n'est pas anodia, ocmme le montre une confrontatice avec les representations śgurbes. Akes que ke motif giomitrique se borme a préfenter la forme pour elk-mime of rythme la lectwe sans la perturber, la figuration deceurne fattention. liornement

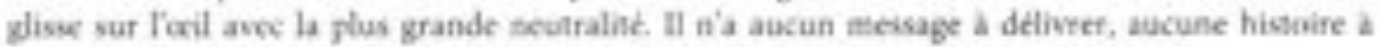
racoenter, ef n'a pas pour volonte premikere de faire refefence a un objet de la nature.

La mature ex, en effet, linspiratrice universelle tant des arts plastiques que des arts de surface. Dans ces deeniers, bien que l'on ait renoncé au relief et que lon ait fait du trasé des contours un ekment de ecpetsentation. Fimitatioe de la nature se fait a travers la linéarité d'un desuin. ples. ou moins abstrait. Comme le notait A. Ricgl. - Derrière toute cruvre dart reide ute production de la nature, soit selle quelle, sank modifcation, wit transobemec par fhomme pur plaisir ou pat

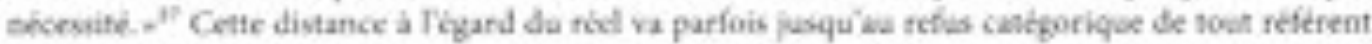
Sgaratif. Ia repecisentation se compose esclusirement de spirales, de lignes continues, de zigrag. típets. Cepsendant, metme ak, de prime abord, ces entités ne semblent pas issues du rél. elles ne se placent pas pour aulant hors du champ de la nature.

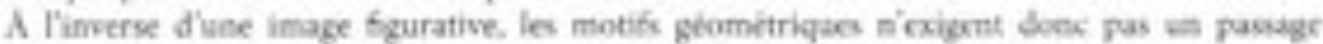
de la viobulite a Vinfriligibulite. Un eencment giomitrigue articonique teste du seul bomaise du vieble. Comme un pociet ea use virgule, loencment sced la lecture ples aisce, par des decors qui

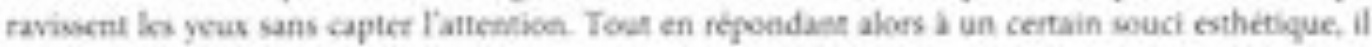
participe a V'intelligbilité de l'écrit, dont it segmeabe le coenenu et indique les pascages importants sans pour atant deconcentrer le lecteur en le detoornant de la coecultatioes da beste. A Foppos. limage figurative interpelle le spectateur, stbra qui clle eveille l'émotion. Celle-ci découle, soit de la reconnaissance de l'objet repriventé, voit de la curiosite aiguike gar Vigatance de la chose ou de la scene roproduites (fige 15 et 16). Contrairement dane image higurative, la répetition des malmes motifs, ares Soemes gitométriques assee simples, n'oblige aucunement le lecteur à s'artéter pour bien

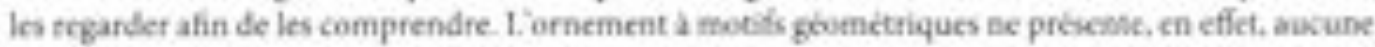

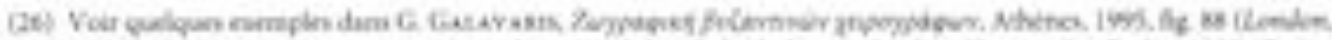

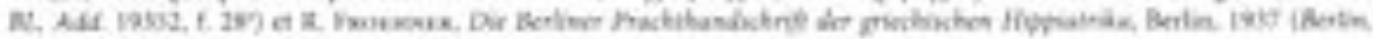

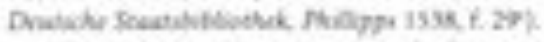

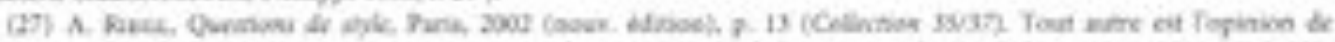

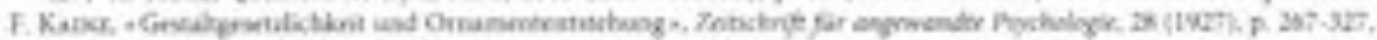

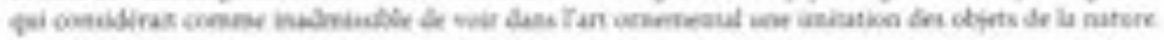




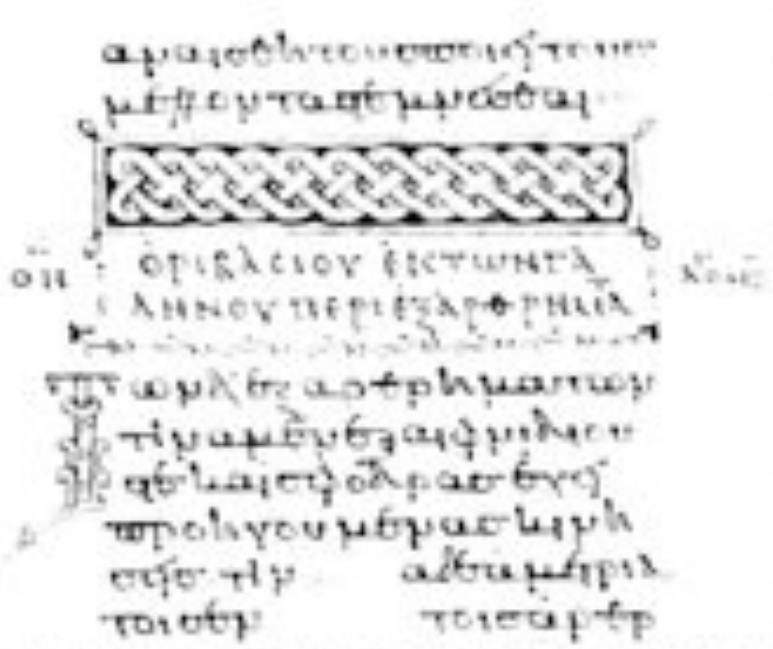

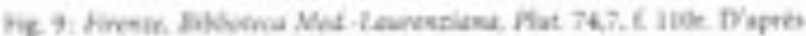

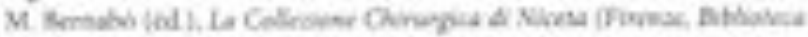

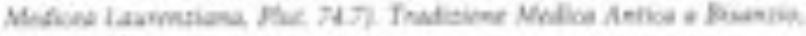
linete. 2000 .

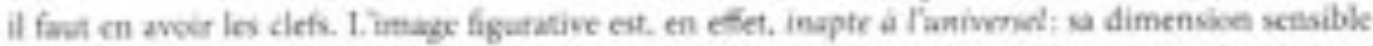
particularise le regard, paralyse la pensete et getne la faculte dabstraction. Use image gtometrique ne vehicule, quant a clik, aucun sens caché, et ciest at ncutralité mekue qui lui confere sa dimension universelle. Oe observe que les mèmes motifs se tencontrest dans dafterentes civilisations, depuis les ipoques les ples reculeos. Fnitin, une fizure est spariaie, eatique. Elle fige la fludite do temps ef n'a ancene capacite dynamique, exobutive. A coetrana. Fart getométrigae, de par ses foemes

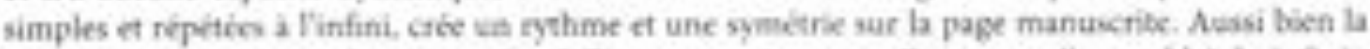

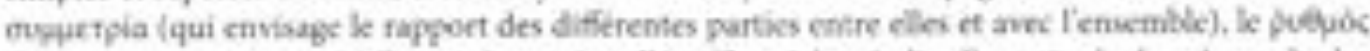

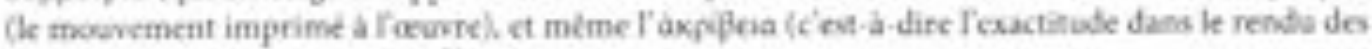
détailbs, sont pris en compte ic"

\section{Ue espace de hihent ou dappartenence?}

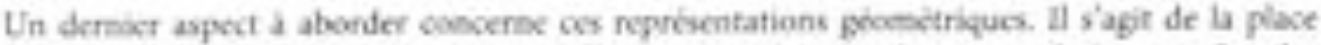
du miniaturiste, ou, sebon certains, du scribe", dans la ralisation de ces mocik. les motifs e los

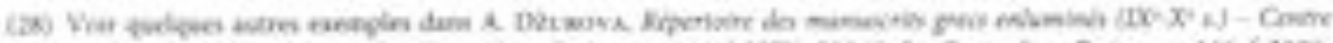

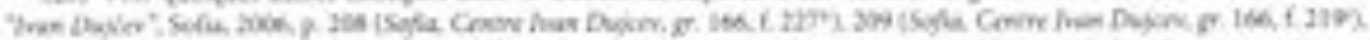

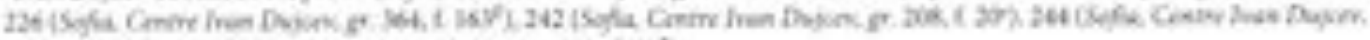

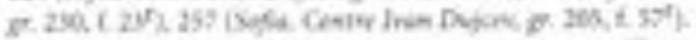

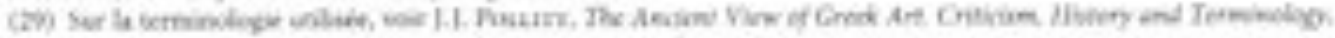

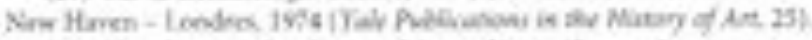

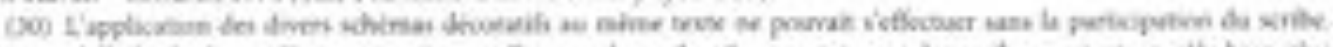

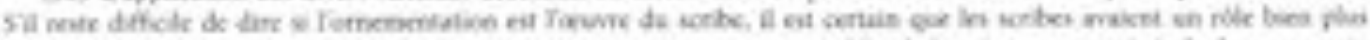

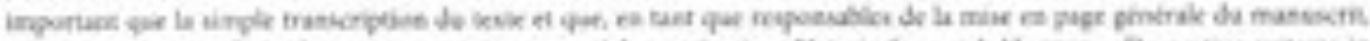

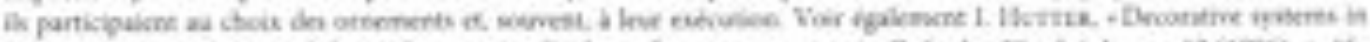

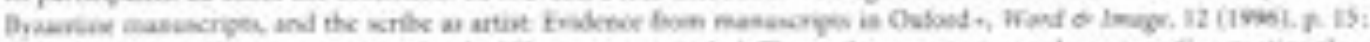

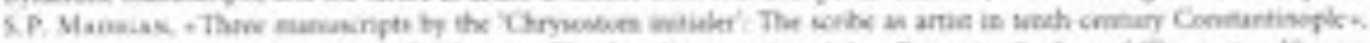

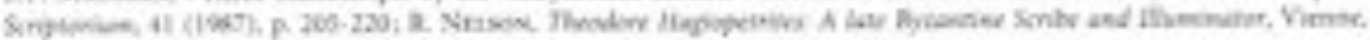
1 wat 

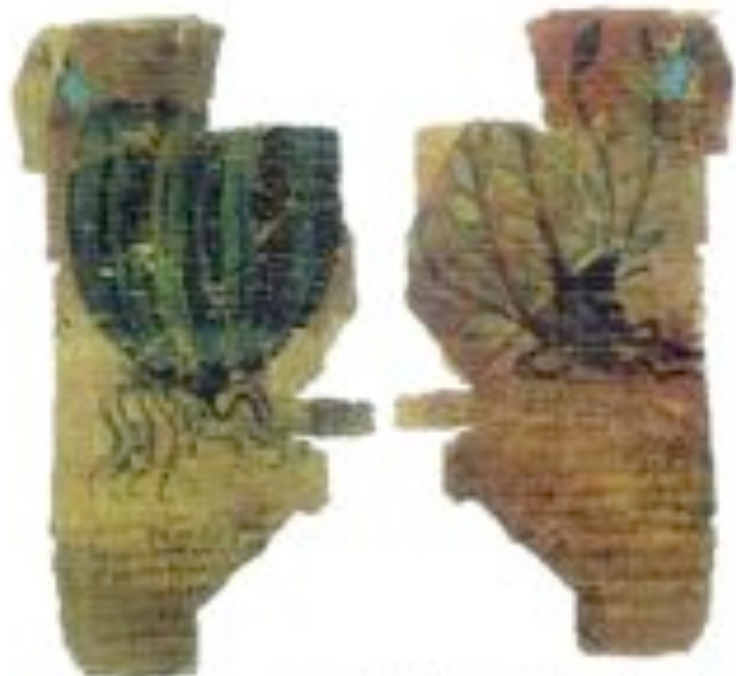

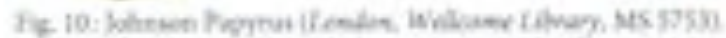

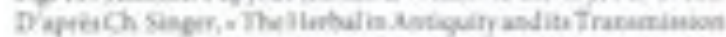

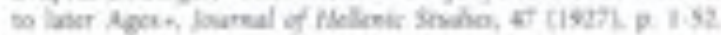

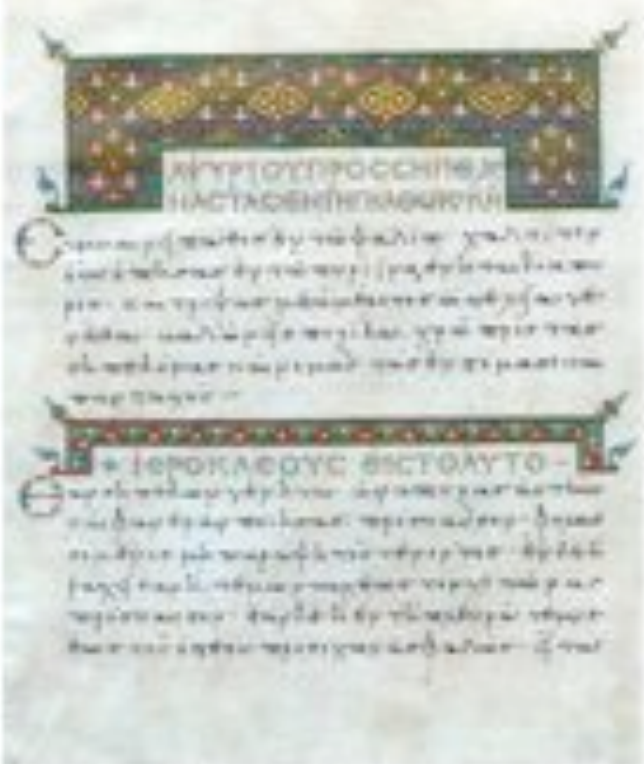

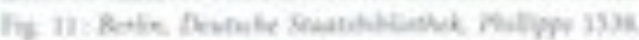

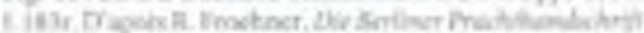

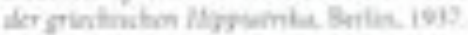

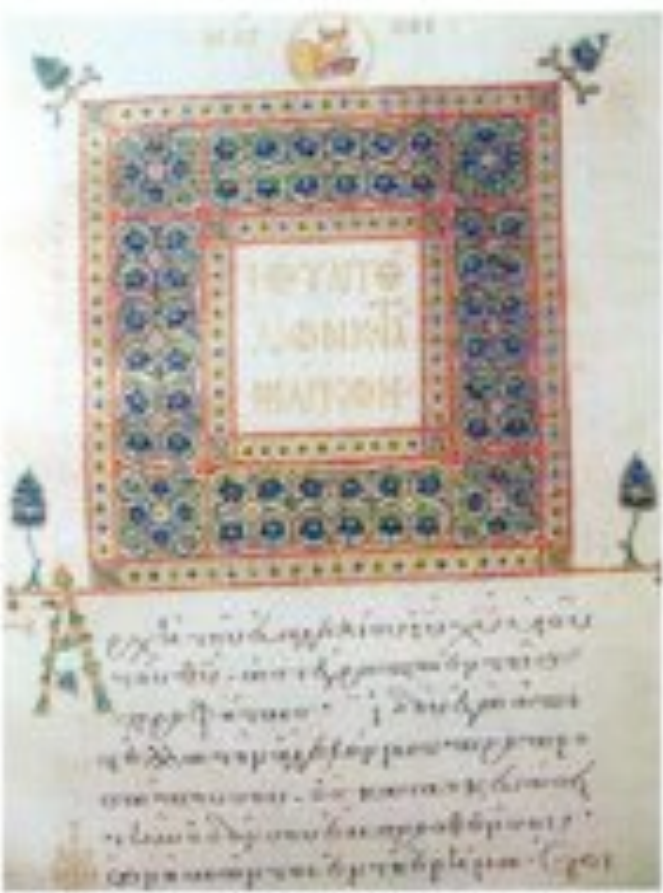

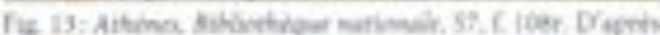

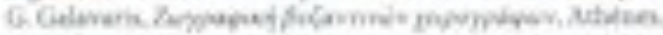
$16 \%, 4 q .9$

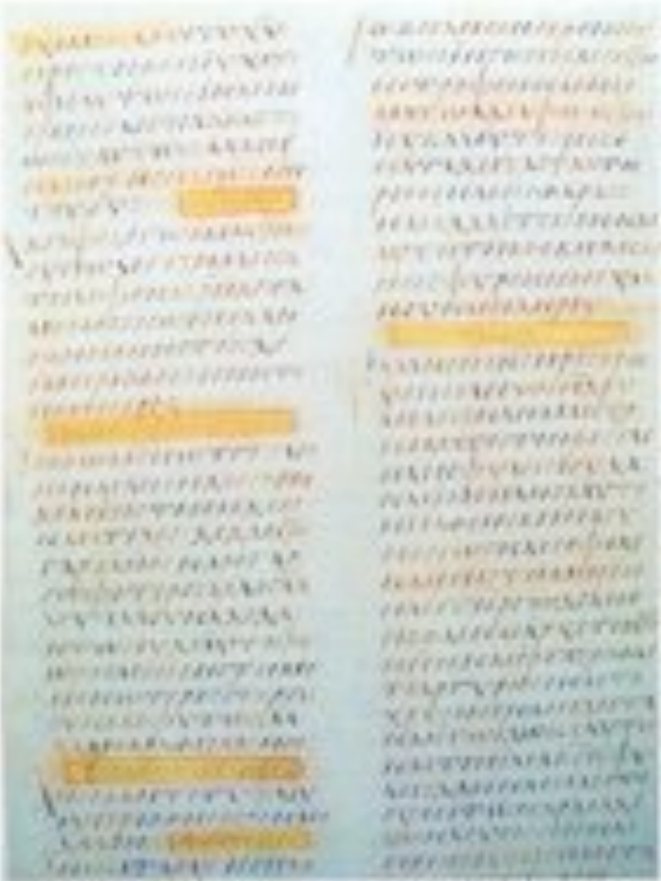

the 12: Sacra Furaliele de Inan Dumucine CPare sNF

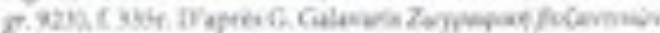

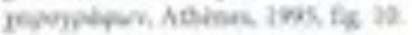




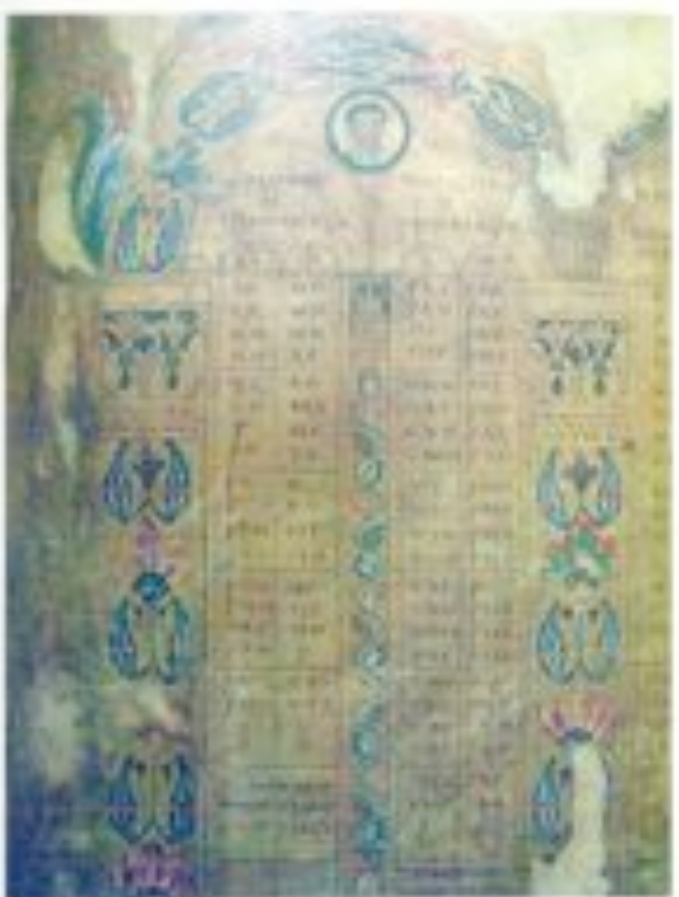

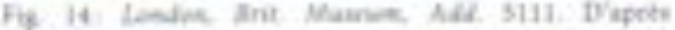

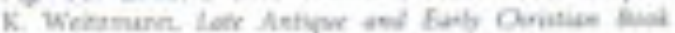

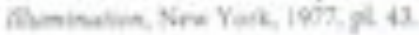

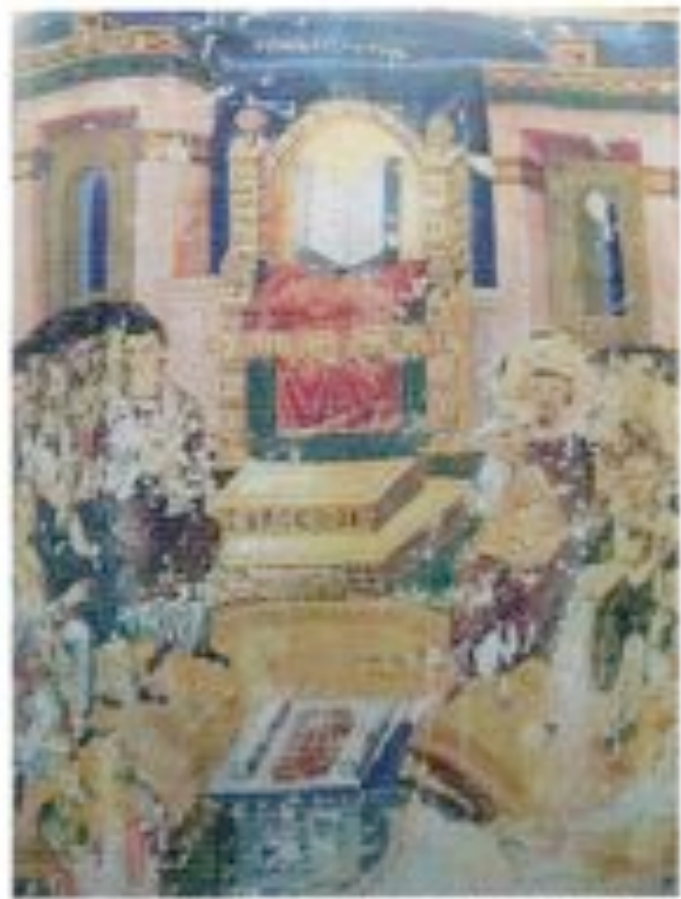

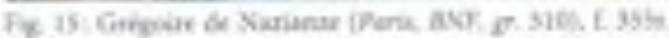

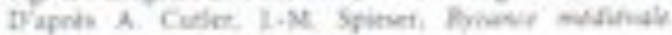

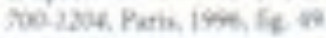

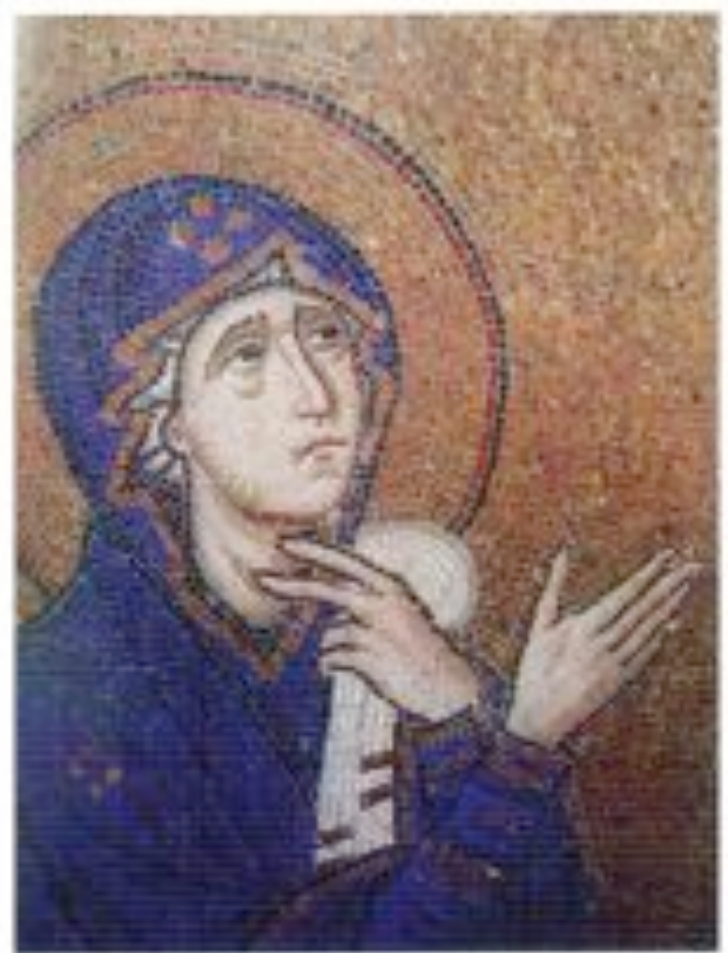

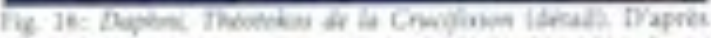

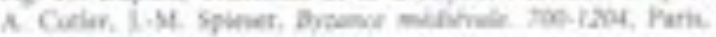
$1 \mathrm{wh}, \mathrm{f}_{4}, 2 \mathrm{w2}$ 
couleurs quit emplote semblent laisser le miniaturiste lbee de ses chois. Ces epaces oreenentaux

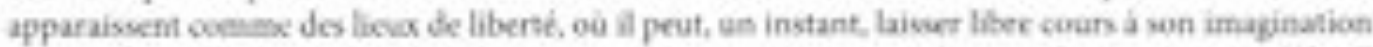

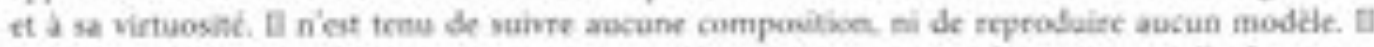

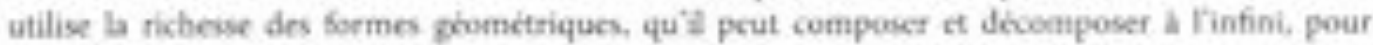
segmester le teste de manictre rézulizte gràce à des motils varies. Ces oempositions sont elles alors comme une litseration de la creiativioe de Thomme byzantia échappant au carcan de la stricte

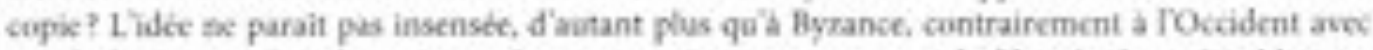
ses Atroleries peuplant les marges des manuserves asown espace de lberté n'et véritablement metnaget. Certes, le miniaturiste peut ne pas vaivre a la lettre le texte a illeister ; il peut tgalement

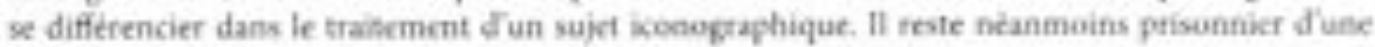
tradition siculaire't,

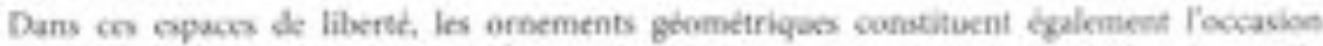
dexprimer use filiation. Au Mogen Age, la notion d'individu tend a se diswoudre dans celle

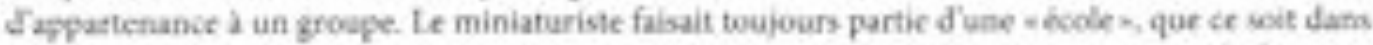

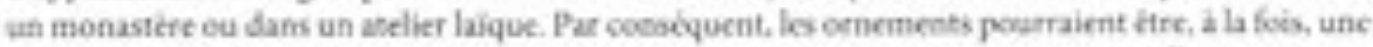
marque personnelle de chajue artiste, unc sorte de sigalure, mais aussi une marqae d'appurtenance

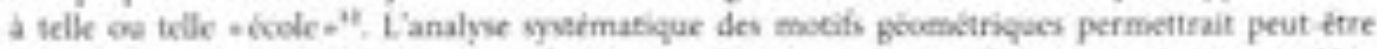
dratteindre ce que les repectentations Siguratives, plas rafes, plus semiles, peinem a montrer. Une tppologie de ces décors ouvrirait noeamment des horizons sur les defërentes - ecoles * omementales.

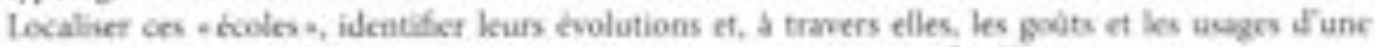

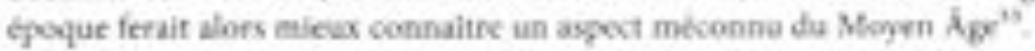

Quel eat, on def-itinc, le states des eencments it moxifs gemetriques dans bs manaszits

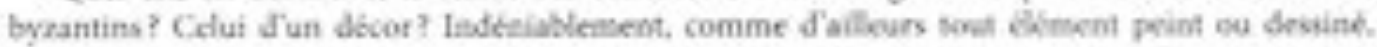

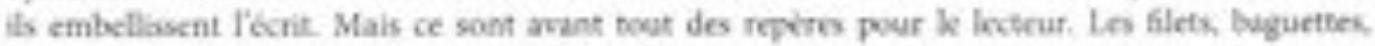
grènetis, ondes, zigages, grecques, entrelacs, volutes et palmettes, pour ne citer que çuelques-urs des motifs conservés dans les manucrits medievaxx, permettest une meilleufe strocturution du

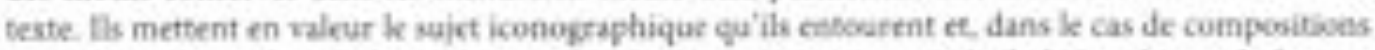

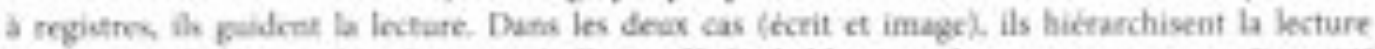
et erienterat le lecteur dans le ceeps de leruvre"t. Probablement plus que tout autre +diepositif

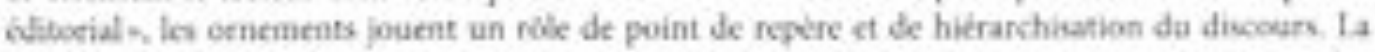
pectetrence accoedte au gotometrigue par rapport au figuratif tient a se neutralite. propre a aider

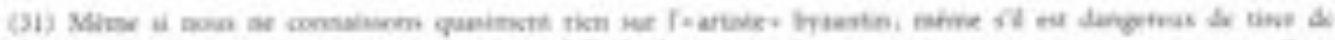

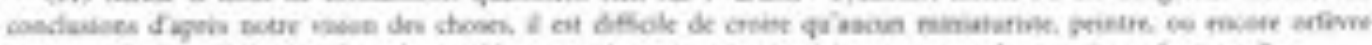

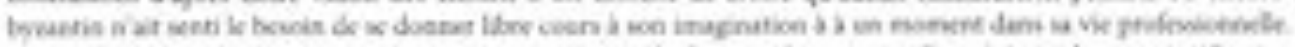

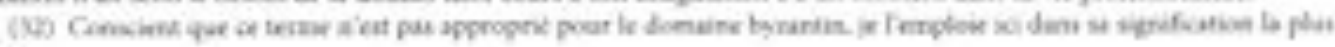
lange

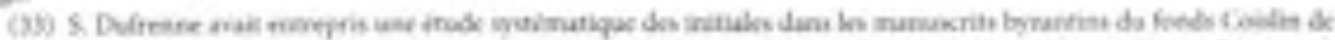

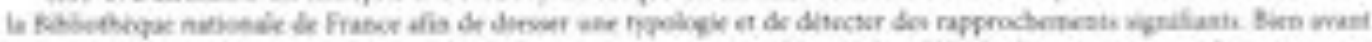

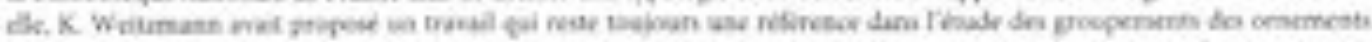

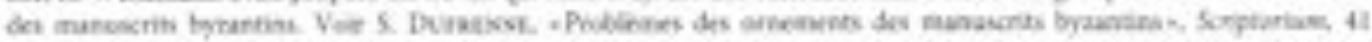

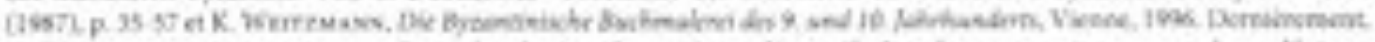

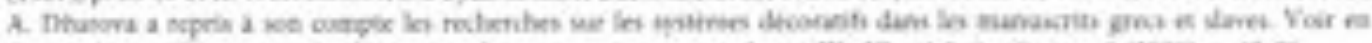

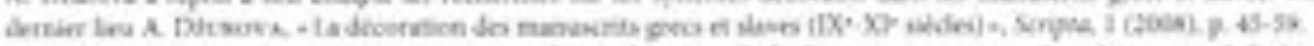

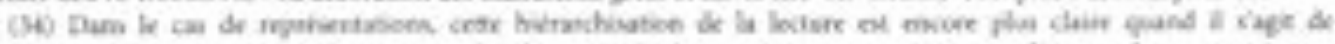

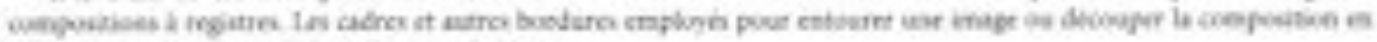

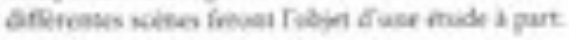


le lecteur sans $k$ perturber, Comme le sulignait D.F. McKenzie, - les formes ont un effet sur le sens *" Et c'cst pour celte raison que le gevmitrique l'a emporti. Autant que de + motif decoranif -

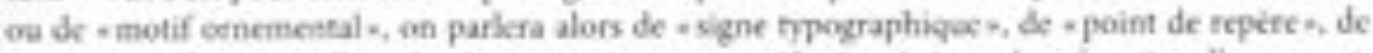

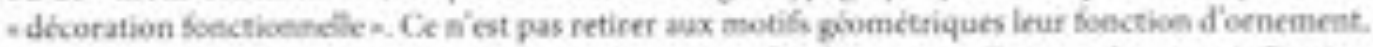

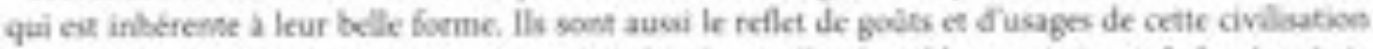

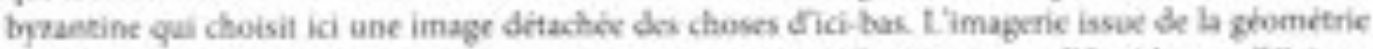
constitue un aspect du tiraillement permancht que coeinait Byance entre Focident at rOtient, entre le figuratif et Taniconique, entre ciel et tefre. 